\title{
Current-Voltage Characteristics of Copper Embedded PtSi/Silicon-p schottky Detector
}

\author{
Seyed Mohammad Hossein Mousakazemi' ${ }^{1}$, Seyedeh Maryam Banihashemian*2, \\ Mehrzad Amiralian ${ }^{3}$, Hassan Hajghassem³ ${ }^{3}$ Seyedeh Habibeh Khaton Banihashemian ${ }^{3}$ \\ 'Department of Basic Sciences, Payame Noor University, Tehran, Iran, smh_mousakazemi@pnu.ac.ir \\ ${ }^{2}$ Department of Physics, Faculty of Science, University of Malaya, 50603 Kuala Lumpur, \\ banihashemian_physics@yahoo.com \\ ${ }^{3}$ Electrical Engineering Department, Islamic Azad University, Tehran, Iran
}

\begin{abstract}
A simple method for developing a Schottky IR sensors consisting of metal (pt)- semiconductor (Si-p) is demonstrated in this work. PtSi/Si-p structure is formed by depositing platinum layer over cleaned Si surface using e-beam and embedded by Copper thin film. Current-voltage (I-V) characterization of PtSi/Si with and without copper is made at $77 \mathrm{~K}$. The I-V measurements are made in the presence and absence of electromagnetic wave with infrared source. The result shows a significant increase in photocurrent and higher sensitivity in copper embedded PtSi/Si structure comparing to the conventional PtSi/Si structure. The CuPt layer created over the PtSi layer increases the PtSi sensitivity by trapping infrared radiation. This sensor with small size and high sensitive for IR radiation can be utilized in infrared imageing sensors, nanoplasmonics and nano-Photonics elements.
\end{abstract}

Keywords: Copper, Current-Voltage, Infrared Detector, PtSi

\section{Introduction}

In the last decades, metal-insulator layer investigations have attracted a lot of interest for scientists because of the surface Plasmon resonance effect. Its application is not only in fiber optic and wave guide but also opened the new application with biomedical scientists and electronic devices. Surface Plasmon resonance (SPR) combine with potential barrier in metal semiconductor interface is a good candidate to enhance the responsibility of infrared detector ${ }^{1-4}$. There are some pioneer research which reported based on SPR in Metal- Semiconductor (MS) interface such as metal-barrier-metal devices and Semiconductor-Insulator-Semiconductor (SIS) heterojunction that have photovoltaic activity from visible to infrared wavelength ${ }^{3}$. Silicide composed in Schottkybarrier structure is a good example in MS candidate as infrared detector ${ }^{2,5}$. PtSi with excellent feature and wide application in detectors and also ohmic contacts attracted a lot of attention for several decades. The work function of PtSi is about $4.97 \mathrm{eV}$ and therefore, it is possible to create a small potential barrier in Silicon-Platinum interface, hence enabled to fabricate high performance photodetector ${ }^{6,7}$. The magnitude of their potential barrier is suitable to absorb infrared light. Platinum silicide are used in infrared imageing sensors for more than two decades. A good quantum efficiency in the silicide detector in infrared region based on Schottky-barrier is defined in the semiconductor industry ${ }^{8-13}$. Mehara et. al. published a research paper on pore size effect of porous silicon on electrical and optical properties of PtSi detectors $^{14}$. Farshid investigated a high sensitivity of schottky junction resulting from $\mathrm{PtSi} /$ porous Si response to 0.8 $1.2 \mathrm{~mm}$ of radiation ${ }^{15}$.

*Author for correspondence 
In the present work, the capability of the PtSi composed embedded with an ultra thin layer of copper is investigated. Experimental characterization of currentvoltage measurement was done on $\mathrm{Cu} / \mathrm{Ptsi} / \mathrm{Si}-\mathrm{P}$ structure in room temperature and liquid nitrogen tmperature. The overall analysis is carried out in terms of dark current and photocurrent. Herein, the most curiosity subjected in potential barrier is proposed by trapping infrared light quanta between $\mathrm{Cu}$ layer and PtSi interface. The performance optimization is carried out, in order to reduce the temperatures effect, to increase dark current and sensitivity.

\section{Experiment}

After RCA and plasma cleaning of p- type silicon, 200 $\mathrm{nm}$ of silicon dioxide is covered on the cleaned silicon surface using e-beam technique. After thermal treatment and annealing about 30 minutes at $500{ }^{\circ} \mathrm{C}$ the sample is ${ }^{\circ} \mathrm{C}$ prepared for photoresist deposition and UV lithography. Eight squares of $1 \mathrm{~mm} \times 1 \mathrm{~mm}$ pattern with UV - mask is used for patterning over silicon dioxide. After developing pattern over the substrate the square area was etched using $\mathrm{Hf}$ solution at controlled time and concentration. Platinum is then deposited on the cleaned silicon surface using e-beam technique, and consequently, a thin layer of copper was deposited on top of platinum surface. By controlling the rate of the deposition, the thickness of the deposited layer was reached to about $50 \mathrm{~nm}$ (Figure 1). Figure 1 shows a sketch image of the fabricated device. The gray color is related to the Aluminum layer which is deposited on the back side and front side of the sample for wire connection. The electrical performance of the fabricated samples was determined by measuring the current (I) as a function of the applied voltage (V) by the HP4145B semiconductor parameter analyzer influenced

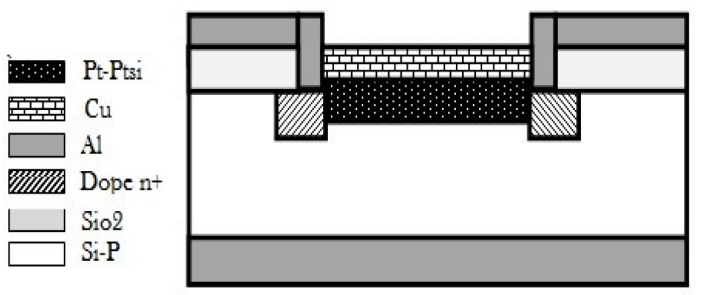

Figure 1. A sketch of fabricated sample, $\mathrm{Cu}-\mathrm{Pt}$ film on a p-type silicon with an ultra-thin insulating ( $\mathrm{SiO} 2)$ layer and array structure. with Infrared lamp. Scanning Electron Microscope (SEM XL30 Philips) is used for analysis of the top layer of the sample.

Figure 2 shows the SEM image of the cross section of the sample which the platinum and copper layers have total thickness of about $50 \mathrm{~nm}$. An X-ray Rutherford back scatter analysis shows the copper, platinum and Silicon peak in the inset. 30 minutes of annealing at $400{ }^{\circ} \mathrm{C}$ is performed in the next stage and an ohmic contact is formed on the front and back side in the final stage.

\section{Result and Discussion}

Figure 3 depicts the current (I) as a function of the applied voltage $(\mathrm{V})$ at liquid nitrogen temperature with and without an infrared lamp exposure. Figure 3 is related by the $\mathrm{PtSi} / \mathrm{Si}$ and Figure 4 is related by Copper embed-

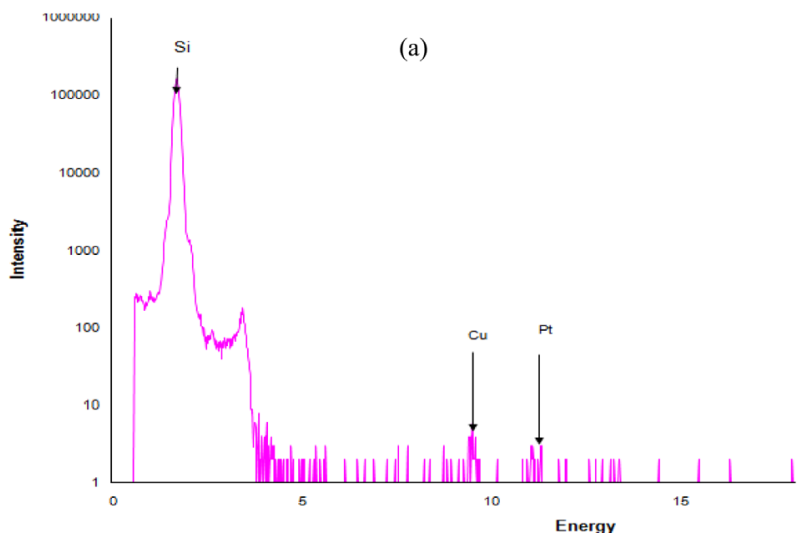

(b)

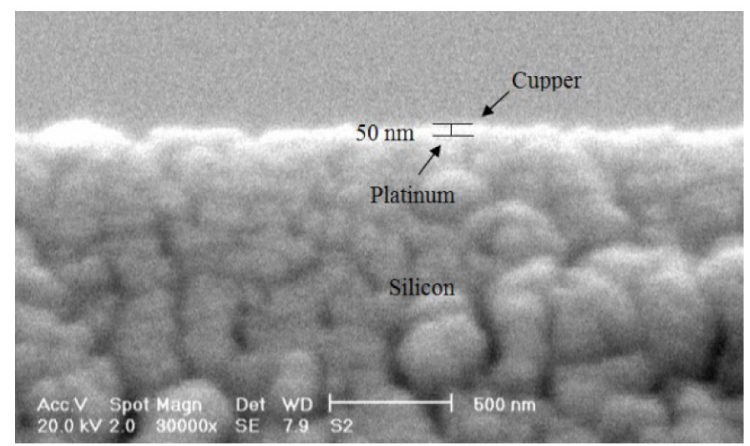

Figure 2. (a) An SEM image of a cross section of Cu-PtSi/ Si multilayer, platinum and copper thin layer have thickness totally about $50 \mathrm{~nm}$. (b) An X-ray Rutherford back scatter analysis, the arrows shows the copper, platinum and Silicon peak positions. 


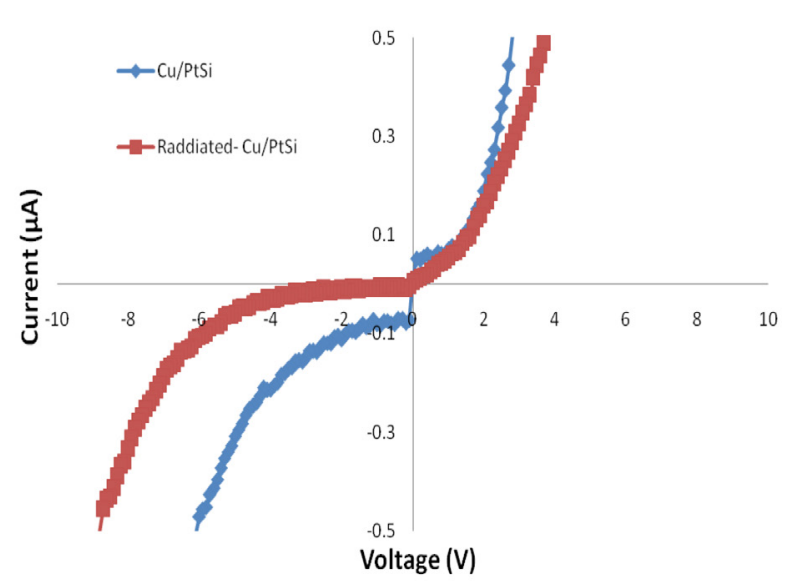

Figure 3. The current (I) as a function of the applied voltage $(\mathrm{V})$ in dark and light situation.

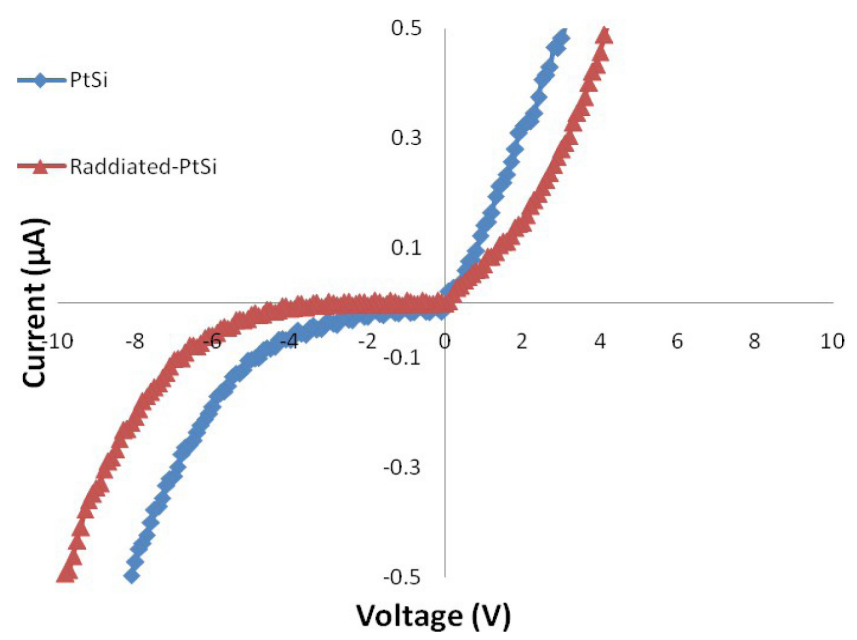

Figure 4. The current (I) as a function of the applied voltage $(\mathrm{V})$ in dark and light situation.

ded the PtSi/Si structure. The current intensity after IR irradiation increased rapidly in copper deposited sample more than the PtSi/Si structure without copper thin film. However, there is a significant increase in the current after light exposure at lower voltages, which is more sensitive comparing to the higher voltages.

Table 1 shows the value of dark current, photo current and the ratio of dark and photo current (LDC) calculated at $77 \mathrm{~K}$ temperature. According to Table 1, the current increases after light exposure in both samples and the LDC is about 8 and 12. The copper embedded PtSi/ $\mathrm{Si} / \mathrm{Al}$ structure act as a resonant cavity and enhances the light absorption efficiency by capturing the light and generating SPR. Thin layer metals multiplies the
Table 1. The dark current, light current and the ratio of dark, light current (LDC) and potential barrier in dark and light status shows respectively in $\mathrm{CuPtSi} / \mathrm{Si}$ structure

\begin{tabular}{lcc}
\hline Parameter & PtSi/Si & Cu-PtSi/Si \\
\hline $\mathrm{I}_{0}=$ Dark Currrent (A) & $1 . \mathrm{E}-09$ & 5.E-09 \\
$\mathrm{I}=$ Photo Current (A) & $8 . \mathrm{E}-09$ & $6 . \mathrm{E}-08$ \\
$\mathrm{LCD}=\mathrm{I} / \mathrm{I}_{0}$ & 8 & 12 \\
\hline
\end{tabular}

reflectance in the interface, as a cavity, and capture the light for a longer time and result to more effectively and therefore, generate more carriers. A density of charge oscillates in copper-platinum interface by absorbing an incident infrared light and propagates on the surface by SPR. By superposition of SPR, the charge carriers obtain sufficient energy and overcome the Schottky barrier and as a result causes the current to be increased rapidly. Due to its better efficiency compare to conventional PtSi/ Si structure, the proposed structure in this work may be a better candidate to be used as a high efficient IR detectors.

\section{Conclusion}

The current - voltage characterization of PtSi nano layer embedded by copper thin film with total thickness of about $50 \mathrm{~nm}$ is carried out. The results indicate that at liquid nitrogen temperature, there is a good response to IR radiation in PtSi embedded by copper compare to convetional PtSi/Si structure. The ratio of photo current to dark current was about 12 which is about 1.5 times larger than $\mathrm{PtSi} / \mathrm{Si}$. It is believed that the increase in the sensitivity of IR detection in this structure is due to capture infrared light and make Plasmon surface resonation at a metal interface causing the enhancement of the carrier energy to overcome the Schottky barrier .

\section{Acknowledgement}

The authors would like to thank Mr. AliAhmadi, for their helpful suggestions and comments. The authors would also like to give special thanks to staff member in electronic Research Centre in Tehran university.

\section{References}

1. H. Elabd, "Theory and Measurements of Photoresponse for Thin Film Pd_2 Si and PtSi Infrared Schottky-Barrier 
Detectors with Optical Cavity," RCA Review, 1982; 143, 569-589.

2. C. Scales, I. Breukelaar, R. Charbonneau, and P. Berini, "Infrared Performance of Symmetric Surface-Plasmon Waveguide Schottky Detectors in Si," Journal of Lightwave Technology, 2011;29, 1852-1860.

3. A. Akbari, R. N. Tait, and P. Berini, "Surface plasmon waveguide Schottky detector," Optics Express, 2010;18, 8505-8514.

4. A. Pradhan, T. Holloway, R. Mundle, H. Dondapati, and M. Bahoura, "Energy harvesting in semiconductor-insulatorsemiconductor junctions through excitation of surface plasmon polaritons," Applied Physics Letters, 2012; 100, 0611271-0611273.

5. S. Zhu, G.-Q. Lo, D.-L. Kwong, H. S. Chu, P. Bai, and E. P. Li, "Silicon Waveguide Infrared Photodiodes Based on Embedded Nickel Silicide Particles," in Optical Fiber Communication Conference, 2012.

6. Y.-C. Lin, K.-C. Lu, W.-W. Wu, J. Bai, L. J. Chen, K. N. Tu, et al., "Single crystalline $\mathrm{PtSi}$ nanowires, $\mathrm{PtSi} / \mathrm{Si} / \mathrm{PtSi}$ nanowire heterostructures, and nanodevices," Nano letters, 2008; 8, 913-918.

7. N. Franco, J. E. Klepeis, C. Bostedt, T. Van Buuren, C. Heske, O. Pankratov, et al., "Experimental and theoretical electronic structure determination for PtSi," Physical Review B, 2003; 68, 045116.

8. J. Yu, X. Zhou, and C. Zhang, "Synthesis of nanoporous PtSi compound by selected dissolution of a Pt 20Si 80 alloy precursor," Materials Letters, 2012; 69, 13-15.

9. H. Hajghassem, A. Erfanian, S. Banihashemian, M. Aliahmadi, and M. Mohtashamifar, "Observation of large magnetic resistance in weak magnetic fields using $\mathrm{CuPt} /$ $\mathrm{SiO}_{2} \mathrm{Si} / \mathrm{SiO}_{2} / \mathrm{CuPt}$ structure," Journal of Magnetism and Magnetic Materials, 2009;321, 2733-2736.

10. H. Hajghassem, S. M. Banihashemian, and M. Aliahmadi, "Low Magnetic Field Detection Using a CuPt Nano Structure Made on a SiO2/Si Structure," Sensors, 2009;9, 9734-9740.

11. S. M. Banihashemian, H. Hajghassem, A. Erfanian, M. Aliahmadi, M. Mohtashamifar, and S. M. Mosakazemi, "Observation and measurement of negative differential resistance on PtSi Schottky junctions on porous silicon," Sensors, 2010;10, 1012-1020.

12. H. Eshghi and M. Mohammadi, "The Effect of Nano And Micro Porosity on The Schottky Barrier Height And Ideality Factor in The I-V Characteristics of Ptsi/P-Si IR Detector," Modern Physics Letters B, 2009;23, 765-771.

13. I. Afandiyeva, S. Demirezen, and Ş. Altındal, "Temperature dependence of forward and reverse bias current-voltage characteristics in Al-TiW-PtSi/n-Si Schottky barrier diodes with the amorphous diffusion barrier," Journal of Alloys and Compounds,2013;552, 423-429.

14. H. Mehrara, A. Erfanian, M. Khaje, M. Zahedinejad, F. Raissi, and F. Rezvani, "Pore size dependence of PtSi/ Porous Si Schottky barrier detectors on quantum efficiency response," Sensors and Actuators A: Physical, 2012; 184, 119-123.

15. K. Kamran, K. Saeid, R. Farshid, and K. Fatemeh, "Highly sensitive porous $\mathrm{PtSi} / \mathrm{Si}$ UV detector with high selectivity," in Micro and Nanoelectronics (RSM), 2013 IEEE Regional Symposium on, 2013, 194-196. 